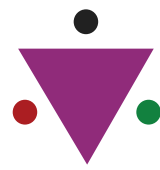

IJCRR Section: Healthcare Sci. Journal Impact Factor: $5.385(2017)$ ICV: 71.54 (2015)

\title{
Are the Re-Emerging Diseases a Real Threat Worldwide?
}

Most of the research worldwide confirm that there is an impending threat to the susceptible population - particularly the children and elderly population, from diseases that are deemed to be controlled but are re appearing again with a vengeance. Studies have shown that re-emerging diseases are a result of multiple risk factors like climate change, social determinants of health, inappropriate drug use, frequent travelling, non - compliance among patients etc. Some studies have elaborated specifically on the dynamic relationship between the infective microorganism, the susceptible host and the favorable environment. They have all predicted a pretty interesting scenario of uncontrolled spread and evolution of the infectious diseases that till now were under barricaded control from a barrage of antimicrobials. Projections of mortality and morbidity due to the impending doom of emerging and re-emerging diseases are a stark reality that is getting harder to combat on a day to day basis. The real problem lies with the use of medications without any standard guidelines, particularly in developing and poor countries. The Global outbreak alert and response network initiated by World Health Organization (WHO) to respond early to any reports of infectious outbreak worldwide. Therefore, coming back to the real problem of uncontrolled existing antibiotic use has resulted in multidrug-resistant pathogens, which is so severe that currently for some infections no new drug is being developed to counter it. Even carbapenems, currently the most successful class of antibiotics are reported to be decreasing in effectiveness. The reason this issue is important from the treatment related point of view is that prevention of diseases among the population takes a long time to show effect. This is true of countries where the actual first line of defense against any infection is early diagnosis and treatment. This is because the healthcare machinery is lagging behind in providing effective primary prevention in terms of vaccination, hygiene, environmental pollution control, effective checks for spread of infectious or exotic diseases either within the country or outside and health education. So along with lack of political will there is a laxity in vision for a disease-free future. Currently, we are not even seriously observing the emerging diseases which along with the reemerging diseases continue to occupy the red alert zone among the health agencies worldwide. Apart from having some organization/organizations to continuously monitor the alerts/outbreaks/pandemics worldwide, the individual countries have an obligation to devise means and methods to organize an effective defense against the re-emergence of diseases in an invincible avatar invulnerable to being taken down effectively. A similar observation voiced by Catharine I Paules et al (2017) in her study, who said that research and public health focus on microbes like Zika virus, West Nile virus, Chikungunya virus etc. to avoid the danger of complacency and failure. Lastly, the threat is real, enormous and will get out of control. Devastation at a global scale would merely be an understatement unless we come together to develop a comprehensive and effective health care plan. 